\title{
Detecting a Stochastic Gravitational-Wave Background: The Overlap Reduction Function
}

\author{
Lee Samuel Finn? \\ Department of Physics, Department of Astronomy and Astrophysics, and Center for Gravitational Wave Physics, \\ The Pennsylvania State University, State College, Pennsylvania, USA 16802-6300 \\ Shane L. Larson \\ Department of Physics, Utah State University, Logan, Utah, USA 84322-4415 \\ Joseph D. Romand用 \\ Department of Physics and Astronomy and Center for Gravitational-Wave Astronomy, \\ The University of Texas at Brownsville, Brownsville, Texas, USA 78520
}

(Dated: September 4, 2018)

\begin{abstract}
Detection of a gravitational-wave stochastic background via ground or space-based gravitationalwave detectors requires the cross-correlation of the response of two or more independent detectors. The cross-correlation involves a frequency-dependent factor - the so-called overlap reduction function or Hellings-Downs curve - that depends on the relative geometry of each detector pair: i.e., the detector separations and the relative orientation of their antenna patterns (beams). An incorrect formulation of this geometrical factor has appeared in the literature, leading to incorrect conclusions regarding the sensitivity of proposed detectors to a stochastic gravitational-wave background. To rectify these errors and as a reference for future work we provide here a complete, first-principles derivation of the overlap reduction function and assess the nature of the errors associated with the use of the incorrect expression that has appeared in the literature. We describe the behavior of the overlap reduction function in different limiting regimes, and show how the difference between the correct and incorrect expressions can be understood physically.
\end{abstract}

PACS numbers: 04.80.Nn, 95.55.Ym, 04.30.-w

\section{INTRODUCTION}

The measured response of a single gravitational-wave detector to a stationary stochastic gravitational-wave signal is indistinguishable from unidentified instrumental noise. The gravitational-wave contribution to the measured response of two or more independent detectors will, however, be correlated between detector pairs in ways that other technical noises will not. The relationship between the power in a stochastic gravitational-wave background and the crosscorrelated response of a detector pair depends on the response of the individual detectors and their relative geometry: i.e., their separation and the relative orientation of their respective detector antenna patterns, or beams. In the context of ground or space-based laser interferometric detectors [1, 2, 3, 4, 5, , 6, 7] or resonant acoustic gravitational-wave detectors [8] this geometrical factor is referred to as the overlap reduction function [9, 10, 11, 12, 13]; in the context of pulsar timing arrays [14] or spacecraft doppler tracking [15] it is called the Hellings-Downs curve [16].

Incorrect expressions for the overlap reduction function have appeared in the recent literature [17, 18] and, with them, incorrect conclusions regarding the sensitivity of proposed gravitational wave detectors to stochastic gravitational waves. These errors have lead to significantly flawed appraisals of the high-frequency sensitivity of the Big Bang Observer to a stochastic gravitational wave background, including spurious nulls in the frequency-dependent detector response and a reduced estimate of the signal-to-noise ratio as a function of the gravitational-wave power. To rectify these errors and as a reference for future work we provide here a complete, first-principles derivation of the overlap reduction function and assess the nature and physical interpretation of the errors associated with the use of the incorrect expression that has appeared in the literature.

\footnotetext{
*Electronic address: LSFinn@PSU.Edu

${ }^{\dagger}$ Electronic address: s.larson@usu.edu

‡Electronic address: joseph.d.romano@gmail.com
} 


\section{THE OVERLAP REDUCTION FUNCTION}

The overlap reduction function of a pair of gravitational-wave detectors is the collection of geometric factors, associated with the relative position and orientation of the detector pair, that appear in the cross-correlation of the detector pair's response. Here we derive an expression for the overlap reduction function by deconstructing the cross-correlation, identifying those contributions that depend only on the radiation and those that depend only on the detectors, which are then identified as the overlap reduction function. This approach has the virtue of clearly illustrating the physical origins of the overlap reduction function and making less likely mistakes of the kind that may have led to the existing errors in the literature.

\section{A. Inter-detector cross-correlation}

Consider two gravitational-wave detectors. A stochastic gravitational-wave "background" will manifest itself in a non-vanishing cross-correlation between the measurements $m(t)$ made at the two detectors, calculated as a time average over the product of the measurements:

$$
C_{T}(\Delta t, t)=\frac{1}{T} \int_{-T / 2}^{T / 2} d t^{\prime} m_{1}\left(t+\Delta t+t^{\prime}\right) m_{2}\left(t+t^{\prime}\right)
$$

The signature of a stochastic gravitational-wave signal $h_{i j}(t, \vec{x})$ is just the expectation value of $C_{T}(\Delta t, t)$ in the presence of $h_{i j}(t, \vec{x})$. Write the measurement $m_{I}(t)$ made at detector $I$ as the sum of a noise contribution $n_{I}(t)$ and a signal contribution $r_{I}(t)$, corresponding to the detector response to $h_{i j}(t, \vec{x})$ [23]. Assume that the noise in each detector is independent and that there are no non-gravitational-wave effects that might lead to a correlation in the measurements made at each detector. Under these assumptions the expectation value of the product $n_{1}(t+\Delta t) n_{2}(t)$ vanishes, implying that the expectation value of $C_{T}(\Delta t, t)$ is just the expectation value of the product $r_{1}(t+\Delta t) r_{2}(t)$ :

$$
\bar{C}(\Delta t) \equiv \overline{C_{T}(\Delta t, t)}=\overline{r_{1}(t+\Delta t) r_{2}(t)}
$$

where overbar denotes expectation value and we have assumed that the expectation value of $C_{T}(\Delta t, t)$ is independent of $t$. This is equivalent to assuming that the background is stationary, as we will describe in more detail in Sec. II C.

Turn now to the detector response. Gravitational waves are weak. Even the most sensitive detectors respond linearly to the local field $h_{i j}(t, \vec{x})$. Correspondingly we write the detector response as a convolution, in time and space, of an impulse response function $R_{I}^{i j}(t, \vec{x})$ with the field $h_{i j}(t, \vec{x})$ :

$$
r_{I}(t) \equiv r_{I}\left(t, \vec{x}_{I}\right)=\int_{-\infty}^{\infty} d \tau \int_{R^{3}} d^{3} x h_{i j}\left(t-\tau, \vec{x}_{I}-\vec{x}\right) R_{I}^{i j}(\tau, \vec{x}),
$$

where $\vec{x}_{I}$ is the spatial location of detector $I$ about which its response $R_{I}^{i j}(t, \vec{x})$ is defined. Causality requires that $R_{I}^{i j}(t, \vec{x})$ vanishes outside the future light cone of $(0, \overrightarrow{0})$. Exploiting the convolution theorem we can also write

$$
r_{I}(t)=(2 \pi)^{3} \int_{-\infty}^{\infty} d f \int_{R^{3}} d^{3} k \widetilde{h}_{i j}(f, \vec{k}) \widetilde{R}_{I}^{i j}(f, \vec{k}) e^{i\left(2 \pi f t-\vec{k} \cdot \vec{x}_{I}\right)}
$$

where

$$
\begin{aligned}
& \widetilde{h}_{i j}(f, \vec{k})=\frac{1}{(2 \pi)^{3}} \int_{-\infty}^{\infty} d t \int_{R^{3}} d^{3} x h_{i j}(t, \vec{x}) e^{-i(2 \pi f t-\vec{k} \cdot \vec{x})} \\
& \widetilde{R}_{I}^{i j}(f, \vec{k})=\frac{1}{(2 \pi)^{3}} \int_{-\infty}^{\infty} d t \int_{R^{3}} d^{3} x R_{I}^{i j}(t, \vec{x}) e^{-i(2 \pi f t-\vec{k} \cdot \vec{x})}
\end{aligned}
$$

are the field Fourier modes and detector transfer function. Note that for real $h_{i j}$ and $R^{i j}$

$$
\begin{aligned}
\widetilde{h}_{i j}^{*}(f, \vec{k}) & =\widetilde{h}_{i j}(-f,-\vec{k}), \\
\widetilde{R}_{I}^{i j *}(f, \vec{k}) & =\widetilde{R}_{I}^{i j}(-f,-\vec{k}) .
\end{aligned}
$$

With the above representations of the detector response, we can express $\bar{C}(\Delta t)$ in terms of the detector response and the field:

$$
\bar{C}(\Delta t)=\int_{-\infty}^{\infty} d \tau \int_{-\infty}^{\infty} d \tau^{\prime} \int_{R^{3}} d^{3} x \int_{R^{3}} d^{3} x^{\prime} \overline{h_{i j}\left(t+\Delta t-\tau, \vec{x}_{1}-\vec{x}\right) h_{k l}\left(t-\tau^{\prime}, \vec{x}_{2}-\vec{x}^{\prime}\right)} R_{1}^{i j}(\tau, \vec{x}), R_{2}^{k l}\left(\tau^{\prime}, \vec{x}^{\prime}\right)
$$


or, equivalently,

$$
\begin{aligned}
& \bar{C}(\Delta t)=(2 \pi)^{6} \int_{-\infty}^{\infty} d f \int_{-\infty}^{\infty} d f^{\prime} \int_{R^{3}} d^{3} k \int_{R^{3}} d^{3} k^{\prime}\left\{e^{2 \pi i\left(f-f^{\prime}\right) t} e^{2 \pi i f \Delta t}\right. \\
& \left.\overline{\tilde{h}_{i j}(f, \vec{k}) \tilde{h}_{k l}^{*}\left(f^{\prime}, \vec{k}^{\prime}\right)}\left[\widetilde{R}_{1}^{i j}(f, \vec{k}) e^{-i \vec{k} \cdot \vec{x}_{1}}\right]\left[\widetilde{R}_{2}^{k l}\left(f^{\prime}, \vec{k}^{\prime}\right) e^{-i \vec{k}^{\prime} \cdot \vec{x}_{2}}\right]^{*}\right\} .
\end{aligned}
$$

Note particularly how the detector location and the transfer function appear together in the combination $\widetilde{R}_{I}^{i j}(f, \vec{k}) e^{-i \vec{k} \cdot \vec{x}_{I}}$. The form of this combination will be critical when we come to understand the physical character of the errors made in earlier calculations of the overlap reduction function.

\section{B. Plane-wave representation of stochastic signal}

Focus attention on gravitational wave fields $h_{i j}(t, \vec{x})$. These are conveniently represented as a superposition of plane waves

$$
h_{i j}(t, \vec{x})=\int_{-\infty}^{\infty} d f \int_{S^{2}} d^{2} \Omega_{\hat{k}} e^{2 \pi i f(t-\hat{k} \cdot \vec{x})} \mathcal{H}_{A}(f, \hat{k}) \mathbf{e}_{i j}^{A}(\hat{k})
$$

where $\hat{k}$ is the unit vector direction of wave propagation, and $\mathbf{e}_{i j}^{A}(\hat{k})$ are the two orthogonal polarization tensors,

$$
\begin{aligned}
& 2 \delta^{A A^{\prime}}=\mathbf{e}_{i j}^{A}(\hat{k}) \mathbf{e}_{i j}^{A^{\prime}}(\hat{k}), \\
& \mathbf{e}_{i j}^{A}(\hat{k})=\mathbf{e}_{j i}^{A}(\hat{k}) .
\end{aligned}
$$

Note that $\mathcal{H}_{A}^{*}(f, \hat{k})=\mathcal{H}_{A}(-f, \hat{k})$ as a consequence of the reality of $h_{i j}$. The plane-wave field amplitudes $\mathcal{H}_{A}(f, \hat{k})$ are related to the field's Fourier modes $\tilde{h}_{i j}(f, \vec{k})$ by

$$
\mathcal{H}_{A}(f, \hat{k}) \mathbf{e}_{i j}^{A}(\hat{k})= \begin{cases}(2 \pi f)^{2} \mathrm{H}_{i j}^{(+)}(f, \hat{k}), & f \geq 0 \\ (2 \pi f)^{2} \mathrm{H}_{i j}^{(-)}(f,-\hat{k}), & f<0\end{cases}
$$

where

$$
\tilde{h}_{i j}(f, \vec{k})=\mathrm{H}_{i j}^{(+)}(f, \hat{k}) \delta(|\vec{k}|-2 \pi f)+\mathrm{H}_{i j}^{(-)}(f, \hat{k}) \delta(|\vec{k}|+2 \pi f)
$$

Here we have introduced separate amplitudes $\mathrm{H}_{i j}^{( \pm)}$for the positive and negative frequency solutions to the dispersion relations $|\vec{k}|^{2}=(2 \pi f)^{2}$ for a plane wave.

Using expansion (2.8), we can write the detector response $r_{I}(t)$ as

$$
r_{I}(t)=\int_{-\infty}^{\infty} d f \int_{S^{2}} d^{2} \Omega_{\hat{k}} \mathcal{H}_{A}(f, \hat{k}) \mathcal{R}_{I}^{A}(f, \hat{k}) e^{2 \pi i f\left(t-\hat{k} \cdot \vec{x}_{I}\right)}
$$

where

$$
\mathcal{R}_{I}^{A}(f, \hat{k})=(2 \pi)^{3} \mathbf{e}_{i j}^{A}(\hat{k}) \widetilde{R}_{I}^{i j}(f, 2 \pi f \hat{k})
$$

The expectation value $\bar{C}(\Delta t)$ can also be written as

$$
\begin{aligned}
& \bar{C}(\Delta t)=\int_{-\infty}^{\infty} d f \int_{-\infty}^{\infty} d f^{\prime} \int_{S^{2}} d^{2} \Omega_{\hat{k}} \int_{S^{2}} d^{2} \Omega_{\hat{k}^{\prime}}\left\{e^{2 \pi i\left(f-f^{\prime}\right) t} e^{2 \pi i f \Delta t}\right. \\
& \left.\overline{\mathcal{H}_{A}(f, \hat{k}) \mathcal{H}_{A^{\prime}}^{*}\left(f^{\prime}, \hat{k}^{\prime}\right)}\left[\mathcal{R}_{1}^{A}(f, \hat{k}) e^{-2 \pi i f \hat{k} \cdot \vec{x}_{1}}\right]\left[\mathcal{R}_{2}^{A^{\prime}}\left(f^{\prime}, \hat{k}^{\prime}\right) e^{-2 \pi i f^{\prime} \hat{k}^{\prime} \cdot \vec{x}_{2}}\right]^{*}\right\} .
\end{aligned}
$$

Again make note of how the detector locations $\vec{x}_{1}, \vec{x}_{2}$ are associated with the respective transfer functions $\mathcal{R}_{1}^{A}$ and $\mathcal{R}_{2}^{A}$. 


\section{Stationarity, isotropy, and polarization correlations}

The statistical properties of the stochastic signal are encoded in the expectation values of products of the gravitational field

$$
\overline{h_{i j}(t, \vec{x})}, \quad \overline{h_{i j}(t, \vec{x}) h_{k l}\left(t^{\prime}, \vec{x}^{\prime}\right)}, \quad \overline{h_{i j}(t, \vec{x}) h_{k l}\left(t^{\prime}, \vec{x}^{\prime}\right) h_{m n}\left(t^{\prime \prime}, \vec{x}^{\prime \prime}\right)}, \cdots
$$

Without loss of generality, we will assume that any non-zero mean has been absorbed in the background spacetime, so that $\overline{h_{i j}(t, \vec{x})}=0$. Furthermore, for Gaussian-distributed fields, knowledge of the quadratic correlations will suffice as all higher-order moments can be constructed from these.

In our problem we expect that the gravitational wave background is effectively stationary: i.e., that $\overline{h_{i j}(t, \vec{x}) h_{k l}\left(t^{\prime}, \vec{x}^{\prime}\right)}$ depends on $t$ and $t^{\prime}$ only through their difference $t-t^{\prime}$. In terms of the plane wave components $\mathcal{H}_{A}(f, \hat{k})$, this condition becomes

$$
\overline{\mathcal{H}_{A}(f, \hat{k}) \mathcal{H}_{A^{\prime}}^{*}\left(f^{\prime}, \hat{k}^{\prime}\right)}=\mathrm{H}_{A A^{\prime}}\left(f, \hat{k}, \hat{k}^{\prime}\right) \delta\left(f-f^{\prime}\right) \text {. }
$$

Thus, the different frequency components of a stationary stochastic background are statistically independent, but they can contribute differently to the cross-correlated power through the $f$-dependence in $\mathrm{H}_{A A^{\prime}}$. Note that the $\delta\left(f-f^{\prime}\right)$ factor in (2.16) eliminates the $t$-dependence in $\bar{C}(\Delta t)$, cf., Eq. 2.14.

If the background is isotropic - i.e., the gravitational-wave specific intensity is independent of the direction of propagation $\hat{k}$ - then the most general form of the quadratic expectation value of the plane wave components $\mathcal{H}_{A}(f, \hat{k})$ is

$$
\overline{\mathcal{H}_{A}(f, \hat{k}) \mathcal{H}_{A^{\prime}}^{*}\left(f^{\prime}, \hat{k}^{\prime}\right)}=\mathrm{H}_{A A^{\prime}}\left(f, f^{\prime}, \hat{k} \cdot \hat{k}^{\prime}\right)
$$

where $\mathrm{H}_{A A^{\prime}}$ depends on $\hat{k}$ and $\hat{k}^{\prime}$ only through the angle between them. If we further assume that the components corresponding to different propagation directions are statistically independent, then

$$
\overline{\mathcal{H}_{A}(f, \hat{k}) \mathcal{H}_{A^{\prime}}^{*}\left(f^{\prime}, \hat{k}^{\prime}\right)}=\mathrm{H}_{A A^{\prime}}\left(f, f^{\prime}\right) \delta^{2}\left(\hat{k}, \hat{k}^{\prime}\right)
$$

where $\delta^{2}\left(\hat{k}, \hat{k}^{\prime}\right) \equiv \delta\left(\cos \theta-\cos \theta^{\prime}\right) \delta\left(\phi-\phi^{\prime}\right)$ is the covariant Dirac delta function on the two-sphere. This latter, more restrictive, condition is the definition of isotropy for gravitational-wave stochastic backgrounds typically assumed in the literature, e.g., [10, 11, 12, 13].

Finally, if the background is unpolarised, by which we will mean that the different polarisation components are statistically independent and contribute equally to the cross-correlated power, then

$$
\overline{\mathcal{H}_{A}(f, \hat{k}) \mathcal{H}_{A^{\prime}}^{*}\left(f^{\prime}, \hat{k}^{\prime}\right)}=\mathrm{H}\left(f, f^{\prime}, \hat{k}, \hat{k}^{\prime}\right) \delta_{A A^{\prime}} .
$$

Putting all these conditions together, we have that an unpolarised, stationary, isotropic stochastic gravitational-wave background satisfies

$$
\overline{\mathcal{H}_{A}(f, \hat{k}) \mathcal{H}_{A^{\prime}}^{*}\left(f, \hat{k}^{\prime}\right)}=\mathrm{H}(f) \delta\left(f-f^{\prime}\right) \delta^{2}\left(\hat{k}, \hat{k}^{\prime}\right) \delta_{A A^{\prime}}
$$

Here $\mathbf{H}(f)$ is a real-valued function proportional to the gravitational-wave energy density. This is, in turn, directly related to $\Omega_{\mathrm{gw}}(f)$, the ratio of the gravitational-wave energy density to the cosmological closure density [13]:

$$
\mathrm{H}(f)=\frac{3 H_{0}^{2}}{32 \pi^{3}} \frac{\Omega_{\mathrm{gw}}(f)}{|f|^{3}},
$$

where $H_{0}$ is the Hubble expansion rate at the present epoch.

\section{The overlap reduction function}

Combining the results of the previous subsections we find that we can express the expectation value $\bar{C}(\Delta t)$ of the inter-detector cross-correlation in the presence of an unpolarised, stationary, isotropic gravitational-wave background as

$$
\bar{C}(\Delta t)=\int_{-\infty}^{\infty} d f e^{2 \pi i f \Delta t} \mathrm{H}(f) \Gamma_{12}(f)
$$


where

$$
\begin{aligned}
\Gamma_{12}(f) & =\int_{S^{2}} d^{2} \Omega_{\hat{k}} \mathcal{R}_{1}^{A}(f, \hat{k}) \mathcal{R}_{2}^{A *}(f, \hat{k}) e^{-2 \pi i f \hat{k} \cdot\left(\vec{x}_{1}-\vec{x}_{2}\right)}, \\
\mathcal{R}_{I}^{A}(f, \hat{k}) & =\mathbf{e}_{i j}^{A}(\hat{k}) \int_{-\infty}^{\infty} d t \int_{R^{3}} d^{3} x R_{I}^{i j}(t, \vec{x}) e^{-2 \pi i f(t-\hat{k} \cdot \vec{x})} .
\end{aligned}
$$

The quantity $\Gamma_{12}(f)$ is the overlap reduction function. It is often convenient to define a normalized overlap reduction function $\gamma_{12}(f) \propto \Gamma_{12}(f)$ with $\gamma_{12}(0)=1$ for two coincident and coaligned identical detectors. For identical interferometers with opening angle $\beta$ this leads to the normalized form

$$
\gamma_{12}(f)=\frac{5}{8 \pi \sin ^{2} \beta} \Gamma_{12}(f) .
$$

Some symmetry properties of $\gamma_{12}(f)$ follow from immediately from its definition: in particular,

$$
\begin{aligned}
& \gamma_{12}^{*}(f)=\gamma_{21}(f), \\
& \gamma_{12}^{*}(f)=\gamma_{12}(-f) .
\end{aligned}
$$

\section{DISCUSSION}

Summarizing the results of the previous section, the overlap reduction function normalized for interferometric detectors is

$$
\gamma_{12}(f)=\frac{5}{8 \pi \sin ^{2} \beta} \int_{S^{2}} d^{2} \Omega_{\hat{k}} \mathcal{R}_{1}^{A}(f, \hat{k}) \mathcal{R}_{2}^{A *}(f, \hat{k}) e^{-2 \pi i f \hat{k} \cdot\left(\vec{x}_{1}-\vec{x}_{2}\right)}
$$

where

$$
\mathcal{R}_{I}^{A}(f, \hat{k})=\mathbf{e}_{i j}^{A}(\hat{k}) \int_{-\infty}^{\infty} d t \int_{R^{3}} d^{3} x R_{I}^{i j}(t, \vec{x}) e^{-2 \pi i f(t-\hat{k} \cdot \vec{x})} .
$$

Adjusting where necessary for differences in the plane-wave expansion and detector numbering conventions, references [17, 18] give the overlap reduction function as

$$
\gamma_{12}^{\prime}(f)=\frac{5}{8 \pi \sin ^{2} \beta} \int_{S^{2}} d^{2} \Omega_{\hat{k}} \mathcal{R}_{1}^{A}(f, \hat{k}) \mathcal{R}_{2}^{A *}(f, \hat{k}) e^{2 \pi i f \hat{k} \cdot\left(\vec{x}_{1}-\vec{x}_{2}\right)} .
$$

Comparing this expression with our Eq. 3.1a we see that they differ by the substitution of $e^{2 \pi i f \hat{k} \cdot\left(\vec{x}_{1}-\vec{x}_{2}\right)}$ for $e^{-2 \pi i f \hat{k} \cdot\left(\vec{x}_{1}-\vec{x}_{2}\right)}$. This difference and when it is significant can be understood physically; doing so provides the occasion for a deeper discussion of the overlap reduction function.

\section{A. Detector locations}

At the end of Sec. IIB we observed that the response of detector $I$, located at $\vec{x}_{I}$, to a field of plane gravitational waves is given by (cf. Eq. 2.12)

$$
r_{I}(t)=\int_{-\infty}^{\infty} d f \int_{S^{2}} d^{2} \Omega_{\hat{k}} \mathcal{H}_{A}(f, \hat{k}) \mathcal{R}_{I}^{A}(f, \hat{k}) e^{2 \pi i f\left(t-\hat{k} \cdot \vec{x}_{I}\right)}
$$

The detector location appears here in the form $e^{-2 \pi i f \hat{k} \cdot \vec{x}_{I}}$. Referring to Eqs. 3.1a and 3.2 for $\gamma_{12}$ and $\gamma_{12}^{\prime}$ it is clear the substitution of $\gamma_{12}^{\prime}$ for $\gamma_{12}$ is equivalent to simply exchanging the locations of detectors 1 and 2 keeping the rest of the configuration of the detectors fixed: i.e., $\gamma_{12}^{\prime}$ is the overlap reduction function for the detector configuration consisting of detector 1 at location $\vec{x}_{2}$ and detector 2 at location $\vec{x}_{1}$. With this understanding we now ask when that exchange is significant and when it is not. 


\section{B. Radiation wavelength and detector separation}

An intuitive understanding of $\gamma_{12}(f)$ recognizes that its behavior in different frequency regimes is governed by several independent dimensionless parameters that can be created from the radiation wavelength, the separation between the detectors, and several intrinsic properties of the detectors as they are represented in the detector impulse response functions.

Referring to Eq. 3.1a we note that when $f\left|\vec{x}_{1}-\vec{x}_{2}\right| \ll 1$ the exponential term may be replaced by unity. As this is the only place where the detector separation appears, in this limit the detector separation plays no role in determining the value or behavior of $\gamma_{12}(f)$. Defining

$$
\delta=f\left|\vec{x}_{1}-\vec{x}_{2}\right|
$$

we refer to $\delta \ll 1$ as the small separation limit. In the small separation limit, then, the difference between $\gamma_{12}$ and $\gamma_{12}^{\prime}$ is negligible under all circumstances.

Now consider the case $\delta \gtrsim 1$. As we have observed, the difference between $\gamma_{12}$ and $\gamma_{12}^{\prime}$ is the difference between locating detector 1 at $\vec{x}_{1}$ or $\vec{x}_{2}$, and detector 2 at $\vec{x}_{2}$ or $\vec{x}_{1}$. When the two detectors are identical in all other aspects, so that $\mathcal{R}_{1}^{A}=\mathcal{R}_{2}^{A}$, this exchange leaves the physical configuration unchanged and, again, there will be no difference between $\gamma_{12}$ and $\gamma_{12}^{\prime}$.

To understand the case $\delta \gtrsim 1$ when the two detectors are not identical we must consider the detector impulse response functions as they appear in Eqs. 3.1.

\section{Radiation wavelength and detector impulse response}

The impulse response of a detector has finite support: i.e., $R_{I}^{i j}(t, \vec{x}) \simeq 0$ for sufficiently large $t>0$ or $|\vec{x}|$. A detector doesn't sample the field beyond its physical extent, so the support in $\vec{x}$ will be on order the detector's size $\ell$. Referring to Eq. 3.1b it is apparent that $\mathcal{R}_{I}^{A}(f, \hat{k})$ depends on $\hat{k}$ only through $\mathbf{e}_{A}^{i j}(\hat{k})$ when $f \ell \ll 1$ :

$$
\mathcal{R}_{I}^{A}(f, \hat{k}) \simeq(2 \pi)^{3} \mathbf{e}_{i j}^{A}(\hat{k}) \tilde{R}_{I}^{i j}(f, \overrightarrow{0}) .
$$

Introducing the parameters

$$
\epsilon_{I}=f \ell_{I}
$$

for the two detectors $I=1,2$ we refer to $\epsilon_{I} \ll 1$ as the small antenna limit for detector $I$.

Return now to the difference between $\gamma_{12}$ and $\gamma_{12}^{\prime}$ when $\delta \gtrsim 1$ and the detectors are not identical. When both $\epsilon_{1}$ and $\epsilon_{2}$ are $\ll 1$ and noting that $\mathbf{e}_{i j}^{A}(-\hat{k})= \pm \mathbf{e}_{i j}^{A}(\hat{k})$ we find

$$
\begin{aligned}
\gamma_{12}(f) & \simeq \frac{5}{8 \pi \sin ^{2} \beta}(2 \pi)^{6} \tilde{R}_{1}^{i j}(f, \overrightarrow{0}) \tilde{R}_{2}^{k l *}(f, \overrightarrow{0}) \int_{S^{2}} d^{2} \Omega_{\hat{k}} \mathbf{e}_{i j}^{A}(\hat{k}) \mathbf{e}_{k l}^{A}(\hat{k}) e^{-2 \pi i f \hat{k} \cdot\left(\vec{x}_{1}-\vec{x}_{2}\right)} \\
& =\frac{5}{8 \pi \sin ^{2} \beta}(2 \pi)^{6} \tilde{R}_{1}^{i j}(f, \overrightarrow{0}) \tilde{R}_{2}^{k l *}(f, \overrightarrow{0}) \int_{S^{2}} d^{2} \Omega_{-\hat{k}} \mathbf{e}_{i j}^{A}(-\hat{k}) \mathbf{e}_{k l}^{A}(-\hat{k}) e^{2 \pi i f \hat{k} \cdot\left(\vec{x}_{1}-\vec{x}_{2}\right)} \\
& =\frac{5}{8 \pi \sin ^{2} \beta}(2 \pi)^{6} \tilde{R}_{1}^{i j}(f, \overrightarrow{0}) \tilde{R}_{2}^{k l *}(f, \overrightarrow{0}) \int_{S^{2}} d^{2} \Omega_{\hat{k}} \mathbf{e}_{i j}^{A}(\hat{k}) \mathbf{e}_{k l}^{A}(\hat{k}) e^{2 \pi i f \hat{k} \cdot\left(\vec{x}_{1}-\vec{x}_{2}\right)} \simeq \gamma_{12}^{\prime}(f)
\end{aligned}
$$

i.e., in the small antenna limit there is no distinction between $\gamma_{12}$ and $\gamma_{12}^{\prime}$.

\section{Large separations and large detectors}

Finally, consider the case $\delta \gtrsim 1$ and, without loss of generality, $\epsilon_{1} \gtrsim 1$. In this case, the variation of the field across the spatial extent of detector 1 is important to the detector response and, in turn to $\gamma_{12}$. Exchanging the detector locations changes the relationship between the spatial extent and orientation of detector 1 relative to the location of detector 2. Correspondingly, in this limit the distinction between $\gamma_{12}$ and $\gamma_{12}^{\prime}$ is significant.

An example of the case $\delta \gtrsim 1, \epsilon_{1}, \epsilon_{2} \gtrsim 1$ is the anticipated sensitivity to a stochastic gravitational-wave background of the Big Bang Observer (BBO), a space-based follow-on to LISA that has been the subject of recent study [7, 17, 19, 20, 21]. The principal results of this study reported in the literature [17, 18] make use of the incorrect form of the overlap reduction function, thus mis-estimating this proposed detector's sensitivity to a stochastic gravitational-wave 

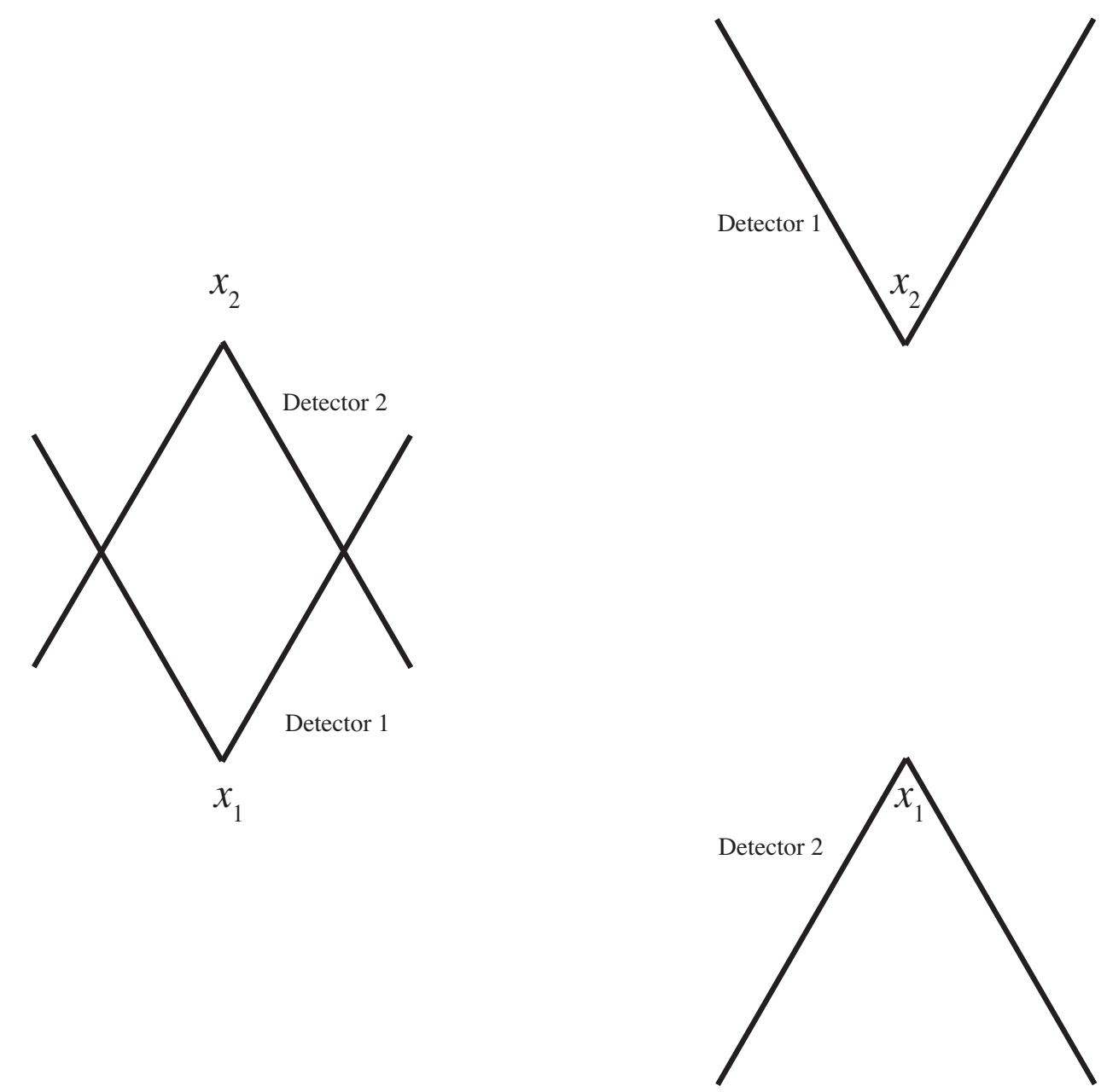

FIG. 1: The arrangement of the two BBO detectors described [17] and the arrangement actually analyzed when $\gamma_{12}^{\prime}$ is substituted for $\gamma_{12}$. Owing to its larger spatial extent, the system actually studied is much more sensitive to frequency than the system whose study was intended.

signal in the higher frequency regime. Figure 1 illustrates the the physical effect of using $\gamma_{12}^{\prime}$ in place of $\gamma_{12}$ when analyzing the cross-correlation of the two BBO detectors as described in [17]. On the left are the two interferometric detectors as they are actually arranged in space; on the right are the effective location and orientation of the detectors when $\gamma_{12}^{\prime}$ is used in place of $\gamma_{12}$ : i.e., when detector 1 is translated to $\vec{x}_{2}$ and detector 2 is translated to $\vec{x}_{1}$. Under this transformation the spatial extent of the two detector pairs is much greater than is actually the case; correspondingly, we expect $\gamma_{12}^{\prime}$ to be a much more sensitive function of frequency than $\gamma_{12}$.

To calculate $\gamma_{12}^{\prime}$ for comparison with $\gamma_{12}$, we need an explicit expression for the transfer function of the detectors. This is derived in [17, Eqs. 5, 7, 11]. In our notation,

$$
\mathcal{R}^{A}(f, \hat{k})=\mathbf{e}_{i j}^{A}(\hat{k}) \frac{1}{2}\left(u^{i} u^{j} \mathcal{T}(\hat{u} \cdot \hat{k}, f)-v^{i} v^{j} \mathcal{T}(\hat{v} \cdot \hat{k}, f)\right),
$$

where

$$
\mathcal{T}(\hat{u} \cdot \hat{k}, f)=\frac{1}{2}\left[\operatorname{sinc}\left(\frac{f}{2 f_{*}}(1-\hat{u} \cdot \hat{k})\right) \exp \left(-i \frac{f}{2 f_{*}}(3+\hat{u} \cdot \hat{k})\right)+\operatorname{sinc}\left(\frac{f}{2 f_{*}}(1+\hat{u} \cdot \hat{k})\right) \exp \left(-i \frac{f}{2 f_{*}}(1+\hat{u} \cdot \hat{k})\right)\right] .
$$

Here $\hat{u}$ and $\hat{v}$ are unit vectors pointing in the direction of the detector arms, $f_{*}=c /(2 \pi L)$ is the transfer frequency of the detectors (arm length $L=5 \times 10^{9} \mathrm{~m}$ ), and $\operatorname{sinc}(x)=\sin (x) / x$. Figure 2 compares $\gamma_{12}^{\prime}$ (cf. [17, Fig. 5]) and $\gamma_{12}$ (Eq. 3.1) for this configuration. As expected $\gamma_{12}^{\prime}$ decreases much more rapidly with frequency and has its nulls more closely spaced than those of $\gamma_{12}$. Figure 3 shows the fractional error $\left|1-\gamma_{12}^{\prime}(f) / \gamma_{12}(f)\right|$ as a function of frequency. The large amplitude spikes in the error occur where nulls of $\gamma_{12}^{\prime}$ do not coincide with nulls of $\gamma_{12}$. 


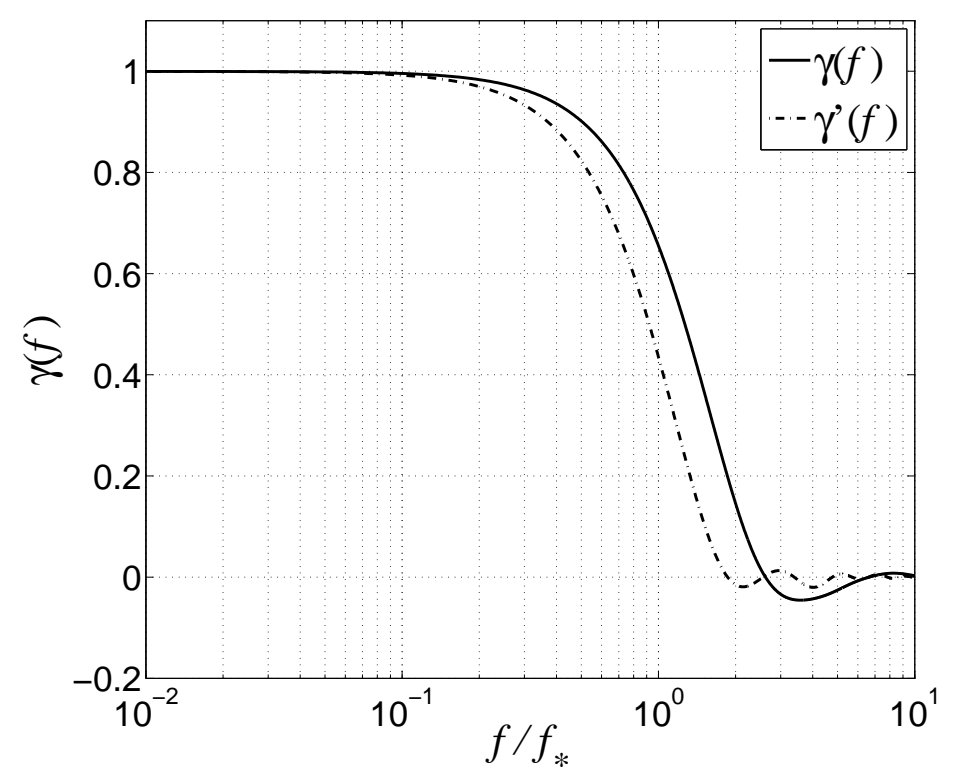

FIG. 2: Plots of the overlap reduction functions $\gamma_{12}(f)$ (solid line) and $\gamma_{12}^{\prime}(f)$ (dot-dashed line) for the BBO configurations in Fig. 1 .

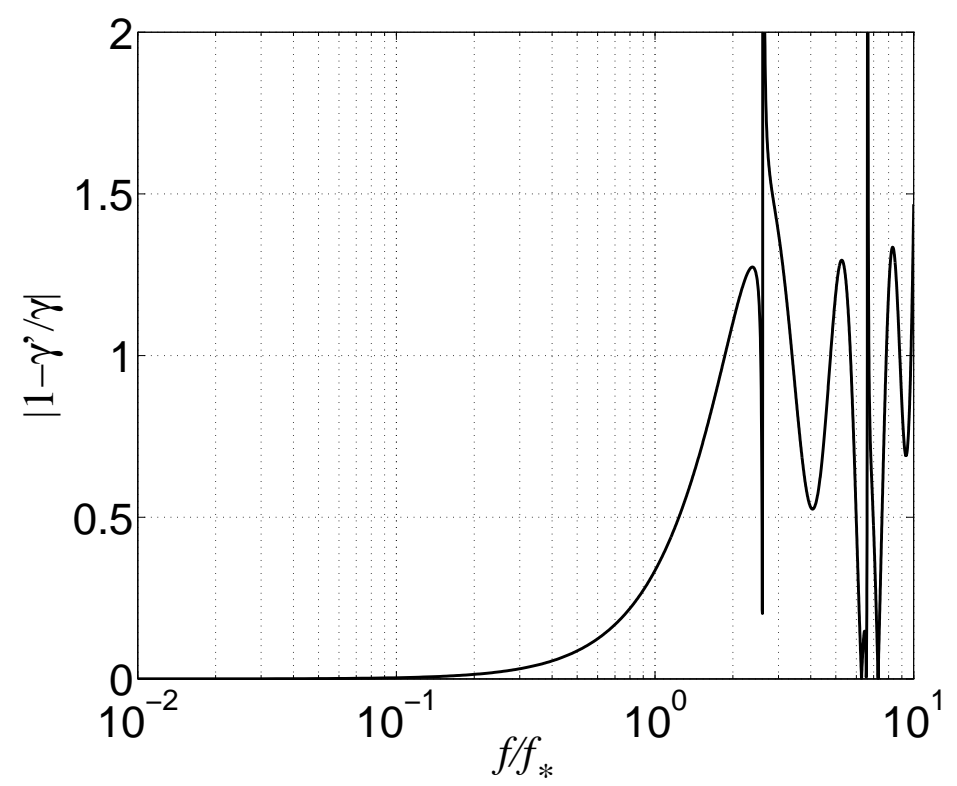

FIG. 3: Fractional difference between $\gamma_{12}(f)$ and $\gamma_{12}^{\prime}(f)$, relative to the correct $\gamma_{12}(f)$ given by Eq. (3.1).

It is apparent from Fig. 2 that the use of an incorrect overlap reduction function has, in this case, led to an underestimate of BBO's sensitivity to a stochastic gravitational wave background. Estimating the background and detector noise power spectral density as white, the magnitude of the error is just the ratio of integrated squared magnitudes $\left|\gamma_{12}(f)\right|^{2}$ and $\left|\gamma_{12}^{\prime}(f)\right|^{2}$,

$$
\frac{\int d f\left|\gamma_{12}^{\prime}(f)\right|^{2}}{\int d f\left|\gamma_{12}(f)\right|^{2}}=1-0.28
$$


i.e., the $\mathrm{BBO}$ estimates of [17, 18] underestimate the sensitivity of $\mathrm{BBO}$ by nearly $30 \%$ across the entire band, and substantially larger if interest is focused on the higher frequencies. Achieving BBO's goals of detecting the stochastic gravitational-wave relics of the inflationary epoch depend on the accurate identification and subtraction of contributions owing to compact binary systems. Underestimating BBO's response to a gravitational-wave background leads to an overestimate of the accuracy required in this identification and subtraction [19]. Recognizing and correcting the underestimate in $\mathrm{BBO}$ sensitivity thus relaxes the analysis problem associated with the identification of these foreground sources.

\section{CONCLUSIONS}

Detection of a gravitational-wave stochastic background relies on the cross-correlated response of one or more pairs of gravitational-wave detectors. The separation and relative orientation of the two detectors plays a crucial role in determining the frequency dependent sensitivity of each detector pair to the stochastic background. Recent studies [17, 18] of the sensitivity of the Big Bang Observer and related future generation gravitational-wave detectors have used an incorrect expression for this geometrical factor. The errors committed may be physically interpreted as an exchange in space of the two detectors, leaving their absolute orientations fixed. In the case of the Big Bang Observer, this error leads to an approximately $30 \%$ underestimate in its sensitivity to relic gravitational waves associated with, e.g., the inflationary epoch. Since achieving BBOs goals of detecting this background requires the accurate identification and subtraction of gravitational-wave foreground contributions from compact binary systems [19], this underestimate has lead to commensurate overestimate of the difficulty of this analysis problem. Recognizing and correcting this error thus improves, in two ways, the prospects for the BBO missions main goal as a Big-Bang Observer.

\section{Acknowledgments}

We most gratefully acknowledge the hospitality of the Aspen Center for Physics and the 2008 Workshop on Gravitational-Wave Astronomy, where most of the work reported here was done. JDR also acknowledges B. Allen, W. Anderson, A. Lazzarini, and J.T. Whelan for discussions. Some of the results in this paper were performed using the HEALPix [22] package (http://healpix.jpl.nasa.gov). The research was supported in part by NSF grant PHY-0555842 awarded to the University of Texas at Brownsville, NSF grant PHY-0653462 and NASA grant NNG05GF71G to The Pennsylvania State University, and the Center for Gravitational Wave Physics, which was supported by the NSF under cooperative agreement PHY-014375.

[1] H. Lück, M. Hewitson, P. Ajith, B. Allen, P. Aufmuth, C. Aulbert, S. Babak, R. Balasubramanian, B. W. Barr, S. Berukoff, et al., Class. Quantum Grav. 23, S71 (2006).

[2] B. C. Barish and R. Weiss, Physics Today 52, 44 (1999).

[3] S. J. Waldman and The LIGO Scientific Collaboration (The LIGO Scientific Collaboration), Class. Quantum Grav. 23, S653 (2006), URL http://stacks .iop.org/0264--9381/23/S653

[4] F. Acernese, P. Amico, M. Alshourbagy, F. Antonucci, S. Aoudia, S. Avino, D. Babusci, G. Ballardin, F. Barone, L. Barsotti, et al., Class. Quantum Grav. 23, S635 (2006), URL http://stacks.iop.org/0264--9381/23/S635

[5] S. Merkowitz and J. C. Livas, eds., Laser Interferometer Space Antenna: Sixth International LISA Symposium, no. 873 in American Institute of Physics Conference Proceedings, Goddard Space Flight Center (American Institute of Physics, Melville, New York, 2007), proceedings of the Sixth International LISA Symposium.

[6] S. Kawamura, M. Ando, T. Nakamura, K. Tsubono, T. Tanaka, I. Funaki, N. Seto, K. Numata, S. Sato, K. Ioka, et al., Journal of Physics: Conference Series 120, 032004 (5pp) (2008), URL http://stacks.iop.org/1742-6596/120/032004

[7] J. Crowder and N. J. Cornish, Physical Review D (Particles, Fields, Gravitation, and Cosmology) 72, 083005 (pages 10) (2005), URL http://link.aps .org/abstract/PRD/v72/e083005.

[8] M. Cerdonio, Class. Quantum Grav. 20, S23 (2003).

[9] P. F. Michelson, Mon. Not. R. Astron. Soc. 227, 933 (1987).

[10] N. Christensen, Phys. Rev. D 46, 5250 (1992).

[11] É. É. Flanagan, Phys. Rev. D 48, 2389 (1993).

[12] B. Allen, in Relativistic Gravitation and Gravitational Radiation, edited by J.-A. Marck and J.-P. Lasota (1997), pp. 373-+.

[13] B. Allen and J. D. Romano, Phys. Rev. D 59, 102001 (1999).

[14] F. A. Jenet, G. B. Hobbs, W. van Straten, R. N. Manchester, M. Bailes, J. P. W. Verbiest, R. T. Edwards, A. W. Hotan, J. M. Sarkissian, and S. M. Ord, Astrophys. J. 653, 1571 (2006), arXiv:astro-ph/0609013.

[15] J. W. Armstrong, Living Reviews in Relativity 9 (2006), URL http://www.livingreviews.org/lrr-2006-1(citedon28Sep2008) 
[16] R. W. Hellings and G. S. Downs, Astrophys. J. Lett. 265, L39 (1983).

[17] N. J. Cornish and S. L. Larson, Class. Quantum Grav. 18, 3473 (2001).

[18] N. Cornish, Class. Quantum Grav. 18, 4277 (2001).

[19] C. Cutler and J. Harms, Physical Review D (Particles, Fields, Gravitation, and Cosmology) 73, 042001 (pages 21) (2006), URL http://link.aps .org/abstract/PRD/v73/e042001

[20] H. Kudoh, A. Taruya, T. Hiramatsu, and Y. Himemoto, Physical Review D (Particles, Fields, Gravitation, and Cosmology) 73, 064006 (pages 16) (2006), URL http://link.aps.org/abstract/PRD/v73/e064006

[21] V. Corbin and N. J. Cornish, Class. Quantum Grav. 23, 2435 (2006).

[22] K. M. Górski, E. Hivon, A. J. Banday, and B. D. Wandelt, Astrophys. J. 622, 759 (2005).

[23] Lower case latin indices $i, j, k, l, \cdots$ will denote spatial components of tensors; upper case latin indices from the middle of the alphabet $I, J, \cdots$ will label different detectors, and upper case latin indices from the beginning of the alphabet $A, A^{\prime}, \cdots$ will denote different gravitational-wave polarizations. 